ORIGINAL ARTICLE

\title{
Serotype prevalence in adults hospitalised with pneumococcal non-invasive community-acquired pneumonia
}

\author{
Thomas Bewick, ${ }^{1}$ Carmen Sheppard, ${ }^{2}$ Sonia Greenwood, ${ }^{1}$ Mary Slack, ${ }^{2}$ \\ Caroline Trotter, ${ }^{3}$ Robert George, ${ }^{2}$ Wei Shen Lim ${ }^{1}$
}

\begin{abstract}
See Editorial, p 473
${ }^{1}$ Department of Respiratory Medicine, Nottingham University Hospitals NHS Trust, Nottingham, UK

${ }^{2}$ Respiratory and Systemic Infection Laboratory, Health Protection Agency, Microbiology Services Division, Colindale, London, UK

${ }^{3}$ School of Social and

Community Medicine, University of Bristol, Bristol, UK
\end{abstract}

\section{Correspondence to}

Dr Thomas Bewick, Department of Respiratory Medicine,

Nottingham University Hospitals NHS Trust, Nottingham NG5 1PB, UK;

thomasbewick@doctors.org.uk

Received 12 September 2011 Accepted 13 January 2012 Published Online First 28 February 2012

\begin{abstract}
Background The distribution of pneumococcal serotypes implicated in non-invasive community-acquired pneumonia (CAP) in adults is currently unknown.

Methods A prospective observational cohort study was conducted over 2 years in a large UK teaching hospital trust. Urine samples, in addition to routine blood and sputum samples, were obtained from consecutive adults admitted to the hospital with CAP. Pneumococcal serotype was determined from urine samples using a validated multiplex immunoassay which detects 14 serotypes.
\end{abstract}

Results Of 920 patients with CAP, 366 had pneumococcal CAP; 242 had a serotype determined. Thirty-day mortality was $10 \%$ for all-cause CAP and $9.6 \%$ for pneumococcal CAP. Annual incidence of pneumococcal CAP was 36.5 per 100000 , increasing from 12.1 to 274.1 per 100000 for ages $16-44$ years and $\geq 85$ years, respectively. The most prevalent serotypes were 14, 1, 8, 3 and 19A. Less invasive serotypes were significantly associated with increasing age (OR per increasing age group: $1.5,95 \% \mathrm{Cl} 1.2$ to 1.9 , $\mathrm{p}<0.001)$ and co-morbidity (OR per increasing Charlson index group: $1.4,95 \% \mathrm{Cl} 1.0$ to $2.0, \mathrm{p}=0.036)$, and with higher 30-day mortality (OR adjusted for age and comorbidity: $5.5,95 \% \mathrm{Cl} 1.2$ to $25.3, \mathrm{p}=0.028$ ) compared with highly invasive serotypes. The proportion of patients in whom serotypes contained within the seven-valent childhood pneumococcal conjugate vaccine was identified increased with age (15.6\% for patients aged $16-44$ years, $41.0 \%$ for patients aged $\geq 85$ years; $\mathrm{p}<0.05)$.

Conclusions In adult invasive and non-invasive pneumococcal CAP, the most common serotypes implicated were 14, 1, 8, 3 and 19A. Age and comorbidity were associated with the distribution of serotypes identified.

\section{INTRODUCTION}

Community-acquired pneumonia (CAP) results in over 100000 admissions to hospitals in England and Wales annually, ${ }^{1}$ and Streptococcus pneumoniae (pneumococcus) is the causative organism in up to $50 \%$ of cases. ${ }^{23}$ The incidence of hospitalised adult CAP in a large European database has been estimated at around 2.75-2.96 per 1000 population, with a steeply increasing incidence with increasing age, and in-hospital mortality of around $14 \% .{ }^{4}$

\section{Key messages}

What is the key question?

- What is the burden of pneumococcal serotypes in non-invasive adult community-acquired pneumonia?

\section{What is the bottom line?}

- The most prevalent pneumococcal serotypes are $14,1,8,3$ and 19A, and while serotype-specific attack rates increase with increasing age and co-morbidity group, this is more pronounced for the less invasive serotypes.

\section{Why read on?}

- This study is the first to describe the serotype distribution in all pneumococcal pneumonia rather than simply invasive disease, and shows that certain serotypes preferentially cause disease in older, frailer adults.

$S$ pneumoniae is surrounded by a polysaccharide capsule, and variation in this capsule allows differentiation into over 90 different serotypes. ${ }^{5}$ The capsule is an important virulence factor, ${ }^{6}{ }^{7}$ and different serotypes exhibit distinct clinical phenotypes, such as invasive potential, ${ }^{8-13}$ and disease outcome. $^{12}{ }^{14-16}$ Serotypes with high invasive potential have been described as "primary pathogens', with serotypes of lower invasiveness described as 'opportunistic pathogens', primarily affecting older patients with underlying co-morbidity. ${ }^{15}$

Seven-, ten-, and thirteen-valent pneumococcal conjugate vaccines (PCV) have been licensed in recent years for use in preventing pneumococcal disease in children (with nine- and eleven-valent vaccines trialled and not licensed, and a fifteenvalent vaccine under development). A seven-valent PCV (PCV-7) was added to UK childhood immunisation schedules in September 2006, and was replaced by PCV-13 in April 2010. Following the introduction of childhood PCV programmes, decreases in vaccine-type serotypes have been seen in invasive pneumococcal disease (IPD) in children, ${ }^{17} 18$ and in adults via a 'herd protection' effect. ${ }^{19} 20$ There is an on-going trial to determine whether vaccinating immunocompetent adults with PCV directly prevents pneumococcal CAP. ${ }^{21}$ In view of the changing pneumococcal disease and 
serotype burden, and with further change predicted, the Joint Committee on Vaccination and Immunisation have recommended that the epidemiology of pneumococcal disease in all age groups be kept under active review. ${ }^{22}$

The majority of studies describing the serotype distribution for pneumococcal CAP have used data derived from blood cultures. ${ }^{11} 121419$ However, blood cultures are positive in only around $5 \%$ of adults with $\mathrm{CAP}^{23}$ and it is not known if data from invasive disease are representative of non-invasive disease. Non-culture methods for determining pneumococcal serotype have a substantially higher sensitivity, ${ }^{24} 25$ but to date there have been no large studies describing the incidence of pneumococcal serotypes in non-invasive CAP.

The aim of the current study is to describe the current burden of pneumococcal CAP in adults particularly in relation to the distribution of pneumococcal serotypes in invasive and noninvasive disease.

\section{METHODS \\ Participants}

Between September 2008 and September 2010 consecutive adult patients (aged $\geq 16$ years) admitted with CAP to two large UK teaching hospitals were prospectively recruited as part of an observational cohort study. These two hospitals serve the population of greater Nottingham with a catchment of approximately 600000 adults. Patients were included if they had at least one acute symptom consistent with a lower respiratory tract infection (breathlessness, cough, sputum or fever), had new infiltrates on chest radiograph and were treated by the admitting team for CAP. Patients were excluded if they had been admitted to hospital in the preceding 10 days, had tuberculosis or had post-obstructive pneumonia due to lung cancer. Participants were identified by investigators on a daily basis from the admitting medical wards and enrolled following informed consent. All patients were managed in a similar manner according to the hospital CAP guidelines at the discretion of the attending clinician. Data related to clinical features, radiological and microbiological investigations and patient outcomes were collected prospectively using a standard proforma. Participants were seen in outpatient clinic 6-8 weeks after discharge to ensure radiographic and clinical resolution, and to ensure that CAP remained the most likely diagnosis. Ethical approval for the study was granted by the Nottingham regional ethics committee.

Urine samples were obtained from each patient at the time of admission. These were transported to the Nottingham University Hospitals Department of Clinical Microbiology, a regional Health Protection Agency (HPA) laboratory, and tested using the Binax NOW immunochromatographic test kit according to the manufacturer's instructions (Binax NOW, Alere Ltd, Stockport, UK). After storage at $-40^{\circ} \mathrm{C}$, urine samples were transported to the Respiratory and Systemic Infection Laboratory (RSIL) at the HPA Microbiology Services Division: Colindale, London, and tested in batches by a multiplexed serotype-specific pneumococcal immunoassay using xMAP beads (Bio-Plex, Bio-Rad, Hercules, California, USA). The assay detects 14 pneumococcal serotypes $(1,3,4,5,6 \mathrm{~A} / \mathrm{C}, 6 \mathrm{~B}, 7 \mathrm{~F} / \mathrm{A}, 8,9 \mathrm{~V}, 14,18,19 \mathrm{~A}, 19 \mathrm{~F}$ and $23 \mathrm{~F})$ with a sensitivity of $79 \% .{ }^{25}$ Serotype was also determined in bacteraemic pneumococcal patients by means of slide agglutination with the latex pool sera purchased from Statens Serum Institut, Copenhagen, Denmark, and the standard group and factor sera at the RSIL, HPA Microbiology Services Division: Colindale, London. Routine blood and sputum samples for bacteriology culture were collected by admitting teams according to the local CAP guidelines and processed in the Nottingham University Hospitals Department of Clinical Microbiology. Culture results were recorded by the study investigators.

\section{Definitions}

Patients were defined as having pneumococcal CAP if any microbiological test was positive for $S$ pneumoniae, including blood culture, sputum culture, Binax NOW, or Bio-Plex serotypespecific antigen detection. Patients with pneumococcal CAP where no serotype was detected after testing with the Bio-Plex assay (ie, $S$ pneumoniae detected by Binax NOW or sputum culture, but no serotype determined by Bio-Plex or blood culture) were described as having 'untyped' pneumococcal disease. Serotypes included in PCV-7 (4, 6B, 9V, 14, 18C, 19F, $23 \mathrm{~F}$ ) are hereafter referred to as 'vaccine-type' (VT), and serotypes not contained within PCV-7 as 'non vaccine-type' (NVT). All VT serotypes are detectable by the Bio-Plex assay. Highly invasive serotypes were defined as $1,5,7 \mathrm{~F}$ and 8 according to previous publications, ${ }^{11-13}$ with the remainder defined as less invasive serotypes.

Annual incidence rates for all-cause and pneumococcal CAP was calculated using the latest census figures (from 2001) for the greater Nottingham area (http://www.nottinghamcity.gov.uk/ index.aspx?articleid=2401). Age group thresholds were the same as those used in the Nottingham census data (16-24, 25-44, $45-64,65-74,75-84$ and $\geq 85$ years). The first two age groups were combined for the purposes of serotype analysis due to the low numbers of participants in both groups. Seasons were defined according to meteorological convention (autumn: September to November; winter: December to February; spring: March to May; summer: June to August). Co-morbidity was as measured using the Charlson co-morbidity index, ${ }^{26}$ grouped into no (score 0$)$, mild $(1-2)$, moderate $(3-4)$ or severe $(5+)$ co-morbidity. ${ }^{1}$

\section{Statistical considerations}

Statistical calculations were made using SPSS V.16.0 (SPSS Inc, 1989-2007). Categorical data were compared using Pearson's $\chi^{2}$, which was also used for univariate analysis and generation of ORs and $95 \%$ CIs. A p value $<0.05$ was taken as statistically significant. A case-control analysis was used to investigate the association between CAP with a less invasive serotype and 30day mortality. Patients who had died within 30 days constituted cases. An a priori conceptual framework based on the previously published data was used to determine potential confounding variables in the multivariable logistic regression. The effect of age and co-morbidity group (as defined above), or disease severity (using the pneumonia severity index $(\mathrm{PSI})^{27}$ ) on the relationship between disease due to a less invasive serotype and 30-day mortality was independently assessed by introducing them into the original model one at a time.

\section{RESULTS}

Of 1099 patients identified with CAP during the study period, 956 consented to be included in the study. Thirty-six patients were unable to provide a urine sample (and had no other test positive for pneumococcus), leaving 920 for analysis. Demographic and outcome data for the cohort are presented in table 1. The median age of the cohort was 71.7 years (IOR 57.8-80.8), and $64.4 \%$ were aged $\geq 65$ years. The 30 -day mortality for the cohort was $10 \%$ ( $9.6 \%$ for patients with pneumococcal CAP). 
Table 1 Demographic data of the study cohort

\begin{tabular}{|c|c|c|}
\hline Patient characteristics & $\begin{array}{l}\text { Whole cohort } \\
(\mathrm{n}=920)\end{array}$ & $\begin{array}{l}\text { Pneumococcal } \\
\text { cohort }(n=366)\end{array}$ \\
\hline \multicolumn{3}{|l|}{ Demographics } \\
\hline Age, median; years (IOR) & $71.7(57.8-80.8)$ & $71.1(52.9-80.7)$ \\
\hline Male (\%) & $546(59.3)$ & $193(52.7)$ \\
\hline $\begin{array}{l}\text { Residential or nursing care } \\
\text { home resident (\%) }\end{array}$ & $51(5.5)$ & $25(6.8)$ \\
\hline WHO performance status $\geq 2(\%)$ & $138(15.0)$ & $62(16.9)$ \\
\hline COPD (\%) & $244(26.5)$ & $94(25.7)$ \\
\hline Ischaemic heart disease (\%) & $145(15.8)$ & $61(16.7)$ \\
\hline Diabetes mellitus (\%) & $130(14.1)$ & $54(14.8)$ \\
\hline Cerebrovascular disease (\%) & $103(11.2)$ & $45(12.3)$ \\
\hline Asthma (\%) & $102(11.1)$ & $47(12.8)$ \\
\hline Congestive cardiac failure (\%) & $74(8.0)$ & $26(7.1)$ \\
\hline Active malignancy (\%) & $67(7.3)$ & $38(10.4)$ \\
\hline Dementia (\%) & $32(3.5)$ & $21(5.7)$ \\
\hline $\begin{array}{l}\text { Mean Charlson co-morbidity } \\
\text { index }(95 \% \mathrm{Cl})\end{array}$ & 1.48 (1.37 to 1.59$)$ & 1.58 (1.40 to 1.76$)$ \\
\hline $\begin{array}{l}\text { Influenza vaccination in } \\
\text { preceding } 12 \text { months (\%) }\end{array}$ & $558 / 855(65.3)$ & $219 / 334(65.6)$ \\
\hline PPV in preceding 10 years (\%) & $383 / 824(46.5)$ & $147 / 320(45.9)$ \\
\hline \multicolumn{3}{|l|}{ Severity } \\
\hline PSI class I-III (\%) & $395(42.9)$ & $151(41.2)$ \\
\hline PSI class IV (\%) & $336(36.5)$ & $132(36.1)$ \\
\hline PSI class V (\%) & $189(20.5)$ & $83(22.7)$ \\
\hline CURB-65 0-1 (\%) & $393(42.7)$ & $143(39.1)$ \\
\hline CURB-65 2 (\%) & $320(34.8)$ & $127(34.7)$ \\
\hline CURB-65 3-5 (\%) & $207(22.5)$ & $96(26.2)$ \\
\hline \multicolumn{3}{|l|}{ Outcome } \\
\hline 30-day mortality (\%) & $92(10.0)$ & $35(9.6)$ \\
\hline LOS & $7(4-12)$ & $8(4-13)$ \\
\hline IRVS (\%) & $82(8.9)$ & $43(11.7)$ \\
\hline
\end{tabular}

COPD, chronic obstructive pulmonary disease; IRVS, invasive respiratory or vasopressor support; LOS, length of (hospital) stay; PPV, pneumococcal polysaccharide vaccine; PSI, pneumonia severity index.

The annual incidence of CAP was 109.8 per 100000 over the 2 years studied, and increased with age from 15.8 per 100000 for ages $16-24$ years to 985.9 per 100000 for those aged $>85$ years (table 2).

Of 366 patients with pneumococcal CAP $(39.8 \%$ of the cohort), a serotype was determined in $242(66.1 \%)$ patients. $S$ pneumoniae was cultured from blood in 40 patients and from sputum or broncho-alveolar lavage in 18 (of which nine were negative by Binax/Bio-Plex), and 196 patients had a positive Binax NOW result. Blood cultures were not taken in 113 patients. For 144 patients in whom Binax NOW testing was negative, a serotype was determined by Bio-Plex.

The distribution of pneumococcal serotypes within the cohort is shown in figure 1 . The most prevalent serotypes were 14

Table 2 The incidence of hospitalised adult community-acquired pneumonia by age group

\begin{tabular}{lccc}
\hline Age group (years) & Population & CAP (n) & $\begin{array}{l}\text { Annual incidence } \\
\text { (per 100 000) }\end{array}$ \\
\hline $16-24$ & 82471 & 26 & 15.8 \\
$25-44$ & 181187 & 115 & 31.7 \\
$45-64$ & 140559 & 140 & 49.8 \\
$65-74$ & 51015 & 295 & 288.4 \\
$75-84$ & 34204 & 300 & 438.5 \\
$85+$ & 11310 & 223 & 985.9 \\
All ages & 500746 & 1099 & 109.7
\end{tabular}

'Population' refers to the number of people in the greater Nottingham area as per the 2001 census. 'Annual incidence' represents the average over the 2 years studied. CAP, community-acquired pneumonia. $(\mathrm{n}=45), 1(\mathrm{n}=40), 8(\mathrm{n}=35), 3(\mathrm{n}=20)$ and 19A $(\mathrm{n}=20)$. Five patients had two serotypes identified; three with serotypes 4 and 8 , one with serotypes $6 \mathrm{~B}$ and $9 \mathrm{~V}$, and one with serotypes $15 \mathrm{~A}$ and 18C. Making the conservative assumption that all patients with untyped pneumococcal CAP had serotypes that were undetectable by Bio-Plex, serotypes contained within PCV13 represented $210(57.4 \%)$ out of 366 patients with pneumococcal CAP, compared with $72(19.7 \%)$ out of 366 patients for PCV-7. The proportion of disease due to PCV-7 VT serotypes increased with age $(7 / 45(15.6 \%)$ for patients aged $16-44$ years when compared with 17/46 (37.0\%) for patients aged 65-74 years and $16 / 39(41.0 \%)$ for patients $>85$ years; $p<0.05$ for both) (table 3).

Admissions to hospital with CAP were more frequent during late autumn and winter, with most admissions between October and January. Pneumococcal disease was more common as a proportion of CAP cases in the winter $(137 / 304,45.1 \%)$ compared with autumn $(89 / 243 ; 36.6 \% ; p=0.047)$, but not significantly different in spring $(82 / 221,37.1 \% ; p=0.068)$ and summer $(58 / 152 ; 38.2 \% ; p=0.160)$. The absolute incidence of pneumococcal infection increased with age, but pneumococcal aetiology was more common as a proportion of CAP cases in younger patients $(47.1 \%$ in patients aged $16-44$ years compared with $34.4 \%$ for age group $65-74$, OR $1.7,95 \%$ CI 1.1 to 2.6 ; $\mathrm{p}=0.020$ ) (table 3). Self-reported influenza and adult pneumococcal vaccination (pneumococcal polysaccharide vaccine (PPV)) rates in patients aged $>65$ years were $433(81.1 \%)$ of 534 and 320 (63.1\%) of 507, respectively. Corresponding values for patients aged $<65$ years were $125(38.9 \%)$ of 321 and $63(19.9 \%)$ of 317 . The odds of pneumococcal pneumonia were not associated with prior vaccination with either PPV (OR 1.0, 95\% CI 0.7 to 1.3; $\mathrm{p}=0.803$ ) or influenza (OR 1.0, 95\% CI 0.8 to $1.4 ; \mathrm{p}=0.881$ ).

The annual age-specific incidences for both highly invasive and less invasive serotypes increased with age (table 4). In a multivariate logistic regression analysis, less invasive serotypes were significantly associated with both increasing age and increasing co-morbidity as measured by the Charlson index (OR per age group 1.5, 95\% CI 1.2 to 1.9, $\mathrm{p}<0.001$; OR per comorbidity group $1.4,95 \%$ CI 1.0 to $2.0, p=0.036$ ). Less invasive serotypes were associated with a higher mortality when compared with highly invasive serotypes after adjustment for both age and co-morbidity group (adjusted OR 5.5, 95\% CI 1.2 to $25.3 ; \mathrm{p}=0.028$ ), or after adjustment for disease severity using PSI group (adjusted OR 8.0, 95\% CI 1.8 to 35.5 , $\mathrm{p}=0.006$ ). Of the 40 patients with pneumococcal bacteraemia, the most prevalent serotype found was $1(n=10)$, followed by 19A $(n=6)$, $8(\mathrm{n}=3), 22 \mathrm{~F}(\mathrm{n}=3)$ and 33F $(\mathrm{n}=3)$. Serotype 14 , the most prevalent serotype in the non-invasive cohort, was not found in bacteraemic patients.

\section{DISCUSSION}

This study is the first, to our knowledge, to describe the pneumococcal serotype distribution in a cohort of adult pneumococcal CAP incorporating both invasive and non-invasive disease. The age-specific incidences for pneumococcal CAP are substantially higher than those suggested elsewhere in CAP database studies. ${ }^{28}$ This is likely to reflect the improved sensitivity of the Binax NOW and Bio-Plex assays over standard culture-based methods. The most common serotypes present within this cohort in descending order of prevalence were 14, 1, 8, 3 and 19A.

The serotype distribution in this cohort of non-invasive disease is broadly similar to the findings in IPD in the UK over 
Figure 1 Serotype distribution of the study cohort. PCV-7 VT: serotype included within the 7-valent pneumococcal conjugate vaccine; PCV-13 VT: serotype included within the 13-valent pneumococcal conjugate vaccine; NVT: non vaccine-type.

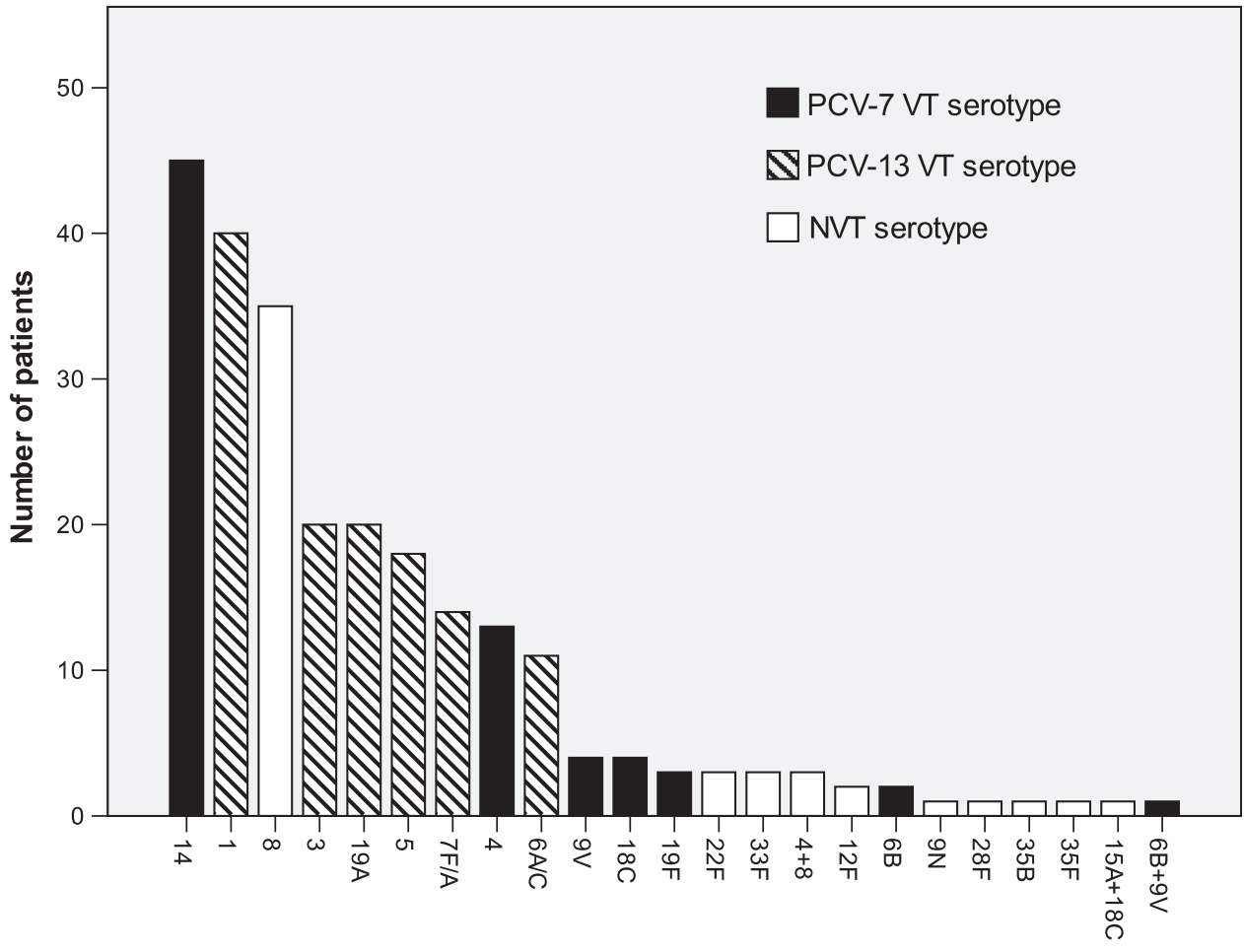

Serotype

a similar time period, ${ }^{19}$ but with a few major differences. For patients aged $\geq 65$ years, the most prevalent serotypes in IPD in the UK between 2008 and 2010 were 19A, 3 and 6A/C, with serotypes 14, 1 and 8 being less frequently seen. Interestingly, serotype 14 , the most prevalent serotype in the current cohort, was not represented within our bacteraemic group. These observations suggest that cohort studies of IPD alone may underestimate the true prevalence of serotype 14. Furthermore, serotype 5 was seen infrequently within a contemporaneous cohort of UK IPD, while it was significantly represented in this cohort of non-invasive disease. Therefore, while IPD cohorts may be used as a partial surrogate for non-invasive disease, certain serotypes may be underestimated by using culture-based methods alone for surveillance.

Falls in VT IPD have been seen in adults within 1 year of the introduction of PCV to child vaccination schedules in both the USA and UK, showing the value of 'herd protection' in adult VT serotype disease. ${ }^{19} 20$ In this study, a PCV-7 serotype was identified in $19.7 \%$ of adults with pneumococcal CAP, compared with $57.4 \%$ with a PCV-13 serotype. Therefore, with the introduction of higher valency PCVs to childhood immunisation schedules, a substantial additional benefit might be realised in adult pneumococcal pneumonia as a result of herd protection.
The fall of VT serotypes in adult IPD has been accompanied by a concomitant rise in some other NVT serotypes, in particular $7 \mathrm{~F}$ and 19A, although there has been a fall in the overall incidence of adult IPD. ${ }^{19}$ Of the serotypes prevalent within this study only serotype 8 is not included in either PCV used in UK childhood vaccination schedules. If the trend towards an increasing proportion of NVT disease continues then this serotype may become substantially more prevalent in the future. It is therefore important to continue to measure the extent of serotype replacement in non-invasive pneumococcal CAP in addition to IPD. ${ }^{29}$

Serotype-specific attack rates increased with increasing age group, but this trend was less marked for highly invasive serotypes compared with less invasive serotypes. This difference is consistent with the concept of highly invasive serotypes acting as 'primary pathogens' which mainly cause disease instead of nasopharyngeal colonisation. ${ }^{15}$ For these serotypes, pathogenrelated factors, such as the thin capsule that highly invasive serotypes possess, ${ }^{12}$ may play a larger role than host factors in the potential to cause disease. By contrast, less invasive serotypes, acting as 'opportunistic pathogens', would be expected to preferentially cause disease in older patients with higher levels of co-morbidity; as observed in this study.

Table 3 The proportion of pneumococcal disease according to age group

\begin{tabular}{|c|c|c|c|c|c|c|}
\hline Age group & $\begin{array}{l}\text { Pneumococcal } \\
\text { CAP }(\%)\end{array}$ & $\begin{array}{l}\text { Annual incidence } \\
\text { (per } 100000)\end{array}$ & VT serotypes (\%) & NVT serotypes (\%) & $\begin{array}{l}\text { OR of VT versus NVT } \\
\text { CAP }(95 \% \text { CI) }\end{array}$ & p Value \\
\hline $16-44(n=136)$ & $64(47.1)$ & 12.1 & $7(10.9)$ & $38(59.4)$ & Reference & - \\
\hline $45-64(n=192)$ & $76(39.6)$ & 27.0 & $14(18.4)$ & 41 (53.9) & $1.9(0.7$ to 5.1$)$ & 0.231 \\
\hline $65-74(n=209)$ & $72(34.4)$ & 70.6 & $17(23.6)$ & $29(40.3)$ & $3.2(1.2$ to 8.7$)$ & 0.024 \\
\hline $75-84(n=242)$ & $92(38.0)$ & 134.5 & $18(19.6)$ & $39(42.4)$ & $2.5(0.9$ to 6.7$)$ & 0.066 \\
\hline $85+(n=141)$ & $62(44.0)$ & 274.1 & $16(25.8)$ & $23(37.1)$ & $3.8(1.4$ to 10.6$)$ & 0.011 \\
\hline All ages $(n=920)$ & $366(39.8)$ & 36.5 & $72(19.7)$ & $170(46.4)$ & - & - \\
\hline
\end{tabular}

CAP, community-acquired pneumonia; NVT, non vaccine-type (serotypes not included within the 7-valent childhood pneumococcal vaccine); VT, vaccine-type (serotypes included within the 7 valent childhood pneumococcal vaccine $(4,6 \mathrm{~B}, 9 \mathrm{~V}, 14,18 \mathrm{C}, 19 \mathrm{~F}, 23 \mathrm{~F})$ ) 
Table 4 Serotype-specific attack rates

\begin{tabular}{lll}
\hline & $\begin{array}{l}\text { Invasive serotypes, } \mathbf{n} \\
\text { (per 100 000 per year) }\end{array}$ & $\begin{array}{l}\text { Less invasive serotypes, } \\
\mathbf{n} \text { (per 100 000 per year) }\end{array}$ \\
\hline $16-44$ & $28(5.3)$ & $17(3.2)$ \\
$45-64$ & $34(12.1)$ & $21(7.5)$ \\
$65-74$ & $17(16.7)$ & $29(28.4)$ \\
$75-84$ & $21(30.7)$ & $36(52.6)$ \\
$85+$ & $7(30.9)$ & $32(141.5)$ \\
\hline
\end{tabular}

Invasive serotypes include 1, 5, 7F and 8 .

PCV in older adults has been suggested as being more effective than the 23-valent PPV in preventing CAP. A trial is currently underway in the Netherlands in unvaccinated older adults $\left(\right.$ CAPITA $^{21}$ ) comparing PCV with placebo in the prevention of pneumococcal disease. The trial should provide valuable data regarding the impact of $\mathrm{PCV}$ on the pneumococcal serotype distribution in this population, and whether there is any additional benefit over and above the 'herd protective' effects resulting from childhood immunisation programmes described herein.

\section{Study limitations}

A proportion (124/366; 33\%) of pneumococcal CAP in this study was labelled as untyped. This may reflect either the presence of serotypes beyond the fourteen detectable by the Bio-Plex assay, or the limited sensitivity of the Bio-Plex assay itself. If the former, then there may be significant NVT serotype(s) that are not being described by this study. The sensitivity of Bio-Plex has been estimated at $79 \%,{ }^{25}$ and therefore a proportion of potentially detectable serotypes will inevitably have been missed. The untyped group were intermediate between VT and NVT serotype groups with regard to 30-day mortality (untyped: $10.8 \%$; VT: $16.7 \%$; NVT: 5.9\%) and co-morbidity (mean Charlson comorbidity index, untyped 1.68; VT 1.97; NVT 1.36). One interpretation of this observation is that the untyped group represents a combination of serotypes from both VT and NVT serotype groups. Alternatively, the serotypes represented in the untyped group may be associated with CAP with different clinical characteristics. Due to the very high specificity of the Bio-Plex assay (>99\%), it is unlikely that a particular serotype has been mis-assigned.

This study was carried out at two large hospitals covering a relatively stable catchment population in Nottingham. As there are no other hospitals in the area, all patients with CAP requiring hospitalisation are admitted to either of the two study hospitals which are part of a single trust and which share the same clinical management protocols. Therefore, selection bias due to differences in hospital admission practices is unlikely. However, as this study included only hospitalised cases, these findings may not apply to patients with CAP treated in the community who have less severe disease.

None of the methods used to define pneumococcal aetiology are perfect diagnostic tests. Blood culture has low sensitivity but high specificity for the detection of pneumococcal disease. ${ }^{23}$ Sputum culture has low sensitivity (predominantly due to difficulties in acquiring an adequate sample), ${ }^{30}$ and may be affected by nasopharyngeal carriage, giving lower specificity. ${ }^{31}$ In this study, only nine patients had a positive pneumococcal sputum culture result without any other positive pneumococcal test. Even if these patients were excluded from the analyses (on the basis that these isolates represented nasopharyngeal carriage alone), the main findings from this study would remain unchanged.
Urinary antigen testing has a sensitivity of around 65\%, higher than conventional culture based techniques. ${ }^{32}$ Nasopharyngeal carriage rather than disease may cause a positive result in children. ${ }^{33}$ However, pneumococcal carriage rates in non-HIV adults from developed countries are generally $<10 \%$ in most cohorts. ${ }^{34} 35$ Additionally, in one study none of the 82 urine samples from adults with confirmed non-pneumococcal CAP registered a serotype using Bio-Plex ${ }^{25}$; in a second study while 24 of 98 adults had evidence of pneumococcal carriage, only three had a positive urinary antigen test. ${ }^{36}$ Therefore, it is likely that in the majority of patients, serotypes identified in this study were implicated in disease (CAP), although we cannot rule out the possibility that in a small proportion of patients the serotypes identified represented nasopharyngeal carriage alone.

\section{CONCLUSIONS}

The most common serotypes implicated in adult invasive and non-invasive pneumococcal CAP are 14, 1, 8, 3 and 19A. Seroepidemiological studies based on IPD cohorts alone may underrepresent certain serotypes important in adult non-invasive pneumococcal disease. Age and co-morbidity were associated with the distribution of serotypes identified.

Acknowledgements The authors thank Christine More and Seyi Eletu from RSIL Colindale, who processed the urine specimens using the Bio-Plex assay, and Robert Cave, Adrian Patrick, Michelle Stannard, Joanne Palfreyman and Andrew Shelton from the Nottingham University Hospitals Microbiology department who processed the urine specimens using the Binax NOW assay. They are also grateful to Tim Harrison from RSIL, Colindale for his support, and Gemma Thompson and Emily Jarvis for their help in recruiting participants. They thank the clinicians and nursing staff of Nottingham University Hospitals NHS Trust, in particular, Melanie Caine, Gaynor Bates and Jo-Anne Harvey, who helped to identify potential participants, and allowed the study investigators to see their patients. The authors are grateful to Alere (formerly Inverness Medical) for manufacturing and providing Binax NOW test kits.

Funding This work was supported by an unrestricted grant from Pfizer, formerly Wyeth. The study was conceived by the authors and the study design was developed and agreed by the authors without any input from the funding body. The study concept and design was peer reviewed by an independent reviewer prior to ethics approval and prior to award of the grant from the funding body. The funding body was not involved in, and had no influence over, study design, data collection, data analyses, interpretation of results, report writing or in the decision to submit the paper for publication. All study data are held solely by the authors. The sponsor for the study was Nottingham University Hospitals NHS Trust.

Competing interests TB and SG have received salaries derived from an unrestricted grant from Wyeth (now Pfizer). CS and MS have received support for travel to meetings for other purposes from Pfizer. MS is on the advisory boards for Wyeth (now Pfizer), Merck and GlaxoSmithKline, and has received grants from GlaxoSmithKline and Pfizer and has also spoken at scientific meetings organised by Pfizer and GlaxoSmithKline. CT has received a grant from the National Institute of Health Research as part of a personal post-doctoral fellowship. RG and WSL have received unrestricted grants from Wyeth (now Pfizer), and $R G$ has received support for travel to meetings for other purposes and grants from Wyeth (now Pfizer) and GlaxoSmithKline.

Ethics approval This study was approved by the Nottingham regional ethics committee.

Provenance and peer review Not commissioned; externally peer reviewed.

\section{REFERENCES}

1. Trotter CL, Stuart JM, George R, et al. Increasing hospital admissions for pneumonia, England. Emerg Infect Dis 2008;14:727-33.

2. Cillóniz C, Ewig S, Polverino E, et al. Microbial aetiology of community-acquired pneumonia and its relation to severity. Thorax 2011;66:340-6. http://dx.doi.org/ 10.1136/thx.2010.143982

3. Lim WS, Macfarlane JT, Boswell TC, et al. Study of community acquired pneumonia aetiology (SCAPA) in adults admitted to hospital: implications for management guidelines. Thorax 2001;56:296-301.

4. Ewig S, Birkner N, Strauss R, et al. New perspectives on community-acquired pneumonia in 388406 patients. Results from a nationwide mandatory performance 
measurement programme in healthcare quality. Thorax 2009:64:1062-9. http://dx. doi.org/10.1136/thx.2008.109785

5. Park IH, Pritchard DG, Cartee R, et al. Discovery of a new capsular serotype (6C) within serogroup 6 of Streptococcus pneumoniae. J Clin Microbiol 2007:45:1225-33. http://dx.doi.org/10.1128/JCM.02199-06

6. Hyams C, Camberlein E, Cohen JM, et al. The Streptococcus pneumoniae capsule inhibits complement activity and neutrophil phagocytosis by multiple mechanisms. Infect Immun 2010;78:704-15. http://dx.doi.org/10.1128/IAl.00881-09

7. Nelson AL, Roche AM, Gould JM et al. Capsule enhances pneumococcal colonization by limiting mucus-mediated clearance. Infect Immun 2007;75:83-90. http://dx.doi.org/10.1128/IAl.01475-06

8. Brueggemann AB, Griffiths DT, Meats $\mathrm{E}$, et al. Clonal relationships between invasive and carriage Streptococcus pneumoniae and serotype- and clone-specific differences in invasive disease potential. J Infect Dis 2003;187:1424-32. http://dx doi.org/10.1086/374624

9. Sandgren A, Sjostrom K, Olsson-Liljequist B, et al. Effect of clonal and serotypespecific properties on the invasive capacity of Streptococcus pneumoniae. J Infect Dis 2004;189:785-96. http://dx.doi.org/10.1086/381686

10. Sleeman KL, Griffiths D, Shackley F, et al. Capsular serotype-specific attack rates and duration of carriage of Streptococcus pneumoniae in a population of children. $J$ Infect Dis 2006;194:682-8. http://dx.doi.org/10.1086/505710

11. Trotter CL, Waight $P$, Andrews NJ, et al. Epidemiology of invasive pneumococcal disease in the pre-conjugate vaccine era: England and Wales, 1996-2006. J Infect 2010;60:200-8. http://dx.doi.org/10.1016/j.jinf.2009.12.008

12. Weinberger DM, Harboe ZB, Sanders EA, et al. Association of serotype with risk of death due to pneumococcal pneumonia: a meta-analysis. Clin Infect Dis 2010;51:692-9. http://dx.doi.org/10.1086/655828

13. Brueggemann AB, Peto TE, Crook DW, et al. Temporal and geographic stability of the serogroup-specific invasive disease potential of Streptococcus pneumoniae in children. J Infect Dis 2004;190:1203-11. http://dx.doi.org/10.1086/423820

14. Luján $\mathbf{M}$, Gallego M, Belmonte $Y$, et al. Influence of pneumococcal serotype group on outcome in adults with bacteraemic pneumonia. Eur Respir J 2010;36:1073-9. http://dx.doi.org/10.1183/09031936.00176309

15. Sjöström K, Spindler C, Ortqvist A, et al. Clonal and capsular types decide whether pneumococci will act as a primary or opportunistic pathogen. Clin Infect Dis 2006:42:451-9. http://dx.doi.org/10.1086/499242

16. Jansen AG, Rodenburg GD, van der Ende A, et al. Invasive pneumococcal disease among adults: associations among serotypes, disease characteristics, and outcome. Clin Infect Dis 2009:49:e23-9. http://dx.doi.org/10.1086/600045

17. Hsu K, Shea K, Stevenson A, et al; Massachusetts Department of Public Health. Changing serotypes causing childhood invasive pneumococcal disease: Massachusetts, 2001-2007. Pediatr Infect Dis J 2010;29:289-93.

18. Techasaensiri C, Messina A, Katz K, et al. Epidemiology and evolution of invasive pneumococcal disease caused by multidrug resistant serotypes of 19A in the 8 years after implementation of pneumococcal conjugate vaccine immunization in Dallas, Texas. Pediatr Infect Dis J 2009. http://dx.doi.org/10.1097/INF.0b013e3181c2a229

19. Miller E, Andrews NJ, Waight PA, et al. Herd immunity and serotype replacement 4 years after seven-valent pneumococcal conjugate vaccination in England and Wales: an observational cohort study. Lancet Infect Dis 2011. http://dx.doi.org/10.1016/ S1473-3099(11)70090-1

20. Hicks LA, Harrison LH, Flannery B, et al. Incidence of pneumococcal disease due to non-pneumococcal conjugate vaccine (PCV7) serotypes in the United States during the era of widespread PCV7 vaccination, 1998-2004. J Infect Dis 2007;196:1346-54. http://dx.doi.org/10.1086/521626
21. Hak E, Sanders EA, Verheij TJ, et al. Rationale and design of CAPITA: a RCT of 13 valent conjugated pneumococcal vaccine efficacy among older adults. Neth $\mathrm{J}$ Med 2008;66:378-83

22. UK Department of Health. JCVI Statement on the Routine Pneumococcal Vaccination Programme for Adults Aged 65 Years and Older. 2011. http://www.dh. gov.uk/prod consum dh/groups/dh digitalassets/@dh/@ab/documents/ digitalasset/dh $1287 \overline{0} 4 . p d f$

23. Campbell SG, Marrie TJ, Anstey R, et al. The contribution of blood cultures to the clinical management of adult patients admitted to the hospital with community-acquired pneumonia: a prospective observational study. Chest 2003;123:1142-50.

24. Leeming JP, Cartwright $\mathrm{K}$, Morris $\mathrm{R}$, et al. Diagnosis of invasive pneumococcal infection by serotype-specific urinary antigen detection. J Clin Microbiol 2005:43:4972-6

25. Sheppard CL, Harrison TG, Smith MD, et al. Development of a sensitive, multiplexed immunoassay using XMAP beads for detection of serotype-specific streptococcus pneumoniae antigen in urine samples. J Med Microbiol 2011;60:49-55. http://dx. doi.org/10.1099/jmm.0.023150-0

26. Charlson ME, Pompei P, Ales KL, et al. A new method of classifying prognostic comorbidity in longitudinal studies: development and validation. J Chronic Dis 1987:40:373-83

27. Fine $\mathbf{M J}$, Auble TE, Yealy DM, et al. A prediction rule to identify low-risk patients with community-acquired pneumonia. N Engl J Med 1997:336:243-50.

28. Melegaro A, Edmunds WJ, Pebody R, et al. The current burden of pneumococcal disease in England and Wales. J Infect 2006;52:37-48. http://dx.doi.org/10.1016/j. jinf.2005.02.008

29. Weinberger DM, Malley R, Lipsitch M. Serotype replacement in disease after pneumococcal vaccination. Lancet 2011. http://dx.doi.org/10.1016/S0140-6736(10) 62225-8

30. Rosón B, Carratalà J, Verdaguer R, et al. Prospective study of the usefulness of sputum Gram stain in the initial approach to community-acquired pneumonia requiring hospitalization. Clin Infect Dis 2000;31:869-74. http://dx.doi.org/10.1086/ 318151

31. Ewig S, Schlochtermeier M, Göke N, et al. Applying sputum as a diagnostic tool in pneumonia: limited yield, minimal impact on treatment decisions. Chest 2002:121:1486-92.

32. Rosón B, Fernández-Sabé N, Carratalà J, et al. Contribution of a urinary antigen assay (Binax NOW) to the early diagnosis of pneumococcal pneumonia. Clin Infect Dis 2004;38:222-6. http://dx.doi.org/10.1086/380639

33. Hamer DH, Egas J, Estrella B, et al. Assessment of the Binax NOW Streptococcus pneumoniae urinary antigen test in children with nasopharyngeal pneumococcal carriage. Clin Infect Dis 2002;34:1025-8. http://dx.doi.org/10.1086/ 339446

34. Flasche S, Van Hoek AJ, Sheasby E, et al. Effect of pneumococcal conjugate vaccination on serotype-specific carriage and invasive disease in England: a crosssectional study. PLoS Med 2011;8:e1001017. http://dx.doi.org/10.1371/journal. pmed. 1001017

35. Spijkerman J, van Gils EJ, Veenhoven RH, et al. Carriage of Streptococcus pneumoniae 3 years after start of vaccination program, the Netherlands. Emerg Infect Dis 2011:17:584-91. http://dx.doi.org/10.3201/eid1704.101115

36. Turner $\mathbf{P}$, Turner $\mathrm{C}$, Kaewcharernnet $\mathrm{N}$, et al. A prospective study of urinary pneumococcal antigen detection in healthy Karen mothers with high rates of pneumococcal nasopharyngeal carriage. BMC Infect Dis 2011;11:108. http://dx.doi. org/10.1186/1471-2334-11-108 